\title{
Modelling, simulation and testing of a reconfigurable cable-based parallel manipulator as motion aiding system
}

\author{
Gianni Castelli and Erika Ottaviano* \\ Laboratory of Robotics and Mechatronics (LARM), University of Cassino, Via Di Biasio 43, 03043 Cassino (Fr), Italy
}

(Received 29 January 2010; final version received 18 June 2010)

\begin{abstract}
This paper presents results on the modelling, simulation and experimental tests of a cable-based parallel manipulator to be used as an aiding or guiding system for people with motion disabilities. There is a high level of motivation for people with a motion disability or the elderly to perform basic daily-living activities independently. Therefore, it is of great interest to design and implement safe and reliable motion assisting and guiding devices that are able to help end-users. In general, a robot for a medical application should be able to interact with a patient in safety conditions, i.e. it must not damage people or surroundings; it must be designed to guarantee high accuracy and low acceleration during the operation. Furthermore, it should not be too bulky and it should exert limited wrenches after close interaction with people. It can be advisable to have a portable system which can be easily brought into and assembled in a hospital or a domestic environment. Cable-based robotic structures can fulfil those requirements because of their main characteristics that make them light and intrinsically safe. In this paper, a reconfigurable four-cable-based parallel manipulator has been proposed as a motion assisting and guiding device to help people to accomplish a number of tasks, such as an aiding or guiding system to move the upper and lower limbs or the whole body. Modelling and simulation are presented in the ADAMS environment. Moreover, experimental tests are reported as based on an available laboratory prototype.
\end{abstract}

Keywords: parallel robots; cable-based systems; assisting devices; experimental robotics

\section{Introduction}

Recent studies show that in Europe the population ratio of senior citizens who are 65 years old exceeds $17 \%$ of the EU-27 population (http://epp.eurostat.ec.europa.eu). Many elderly people cannot perform normal daily householdrelated work because of decreases in the force-generating capacity of their body. Furthermore, a large number of elderly or people with motion disabilities, who stay at home, cannot perform daily life without the aid of other people. An assisting device can help an end-user to perform exercises in a rehabilitation therapy as support for a physiotherapist or basic daily activities such as standing, walking and sitting. The first one is the most crucial operation, since a person with reduced motion capability and physical strength can experience falling, which represents the most serious problem associated with aging.

Several robot prototypes have been proposed in order to explore the feasibility of robots aiding medical personnel. In general, a robot for a medical application should be able to interact with a patient in safety conditions, i.e. it must not damage people or surroundings; it must be designed to guarantee high accuracy and low acceleration during the operation. Furthermore, it should not be too bulky and it should exert limited wrenches after close interaction with people.

It is advisable to have a portable or deployable system which can be easily brought into a hospital or a domestic environment. Cable-based robotic structures can fulfil all these requirements because of their main characteristics that make them light and intrinsically safe.

Cable-based parallel manipulators are robotic systems in which cables are actuated. Cables are connected to the end-effector and a fixed frame through external connectors (Hiller et al. 2005). A cable-based manipulator can operate the end-effector by changing the cables' lengths while preventing any cables becoming slack (Verhoeven et al. 1998). Therefore, feasible tasks are limited to the main static or dynamic characteristics of the cables; because they can only pull the end-effector, but not push it. Furthermore, the cables' tension must be bounded to avoid excessive forces, which may cause stress deformation or failure in the cables (Riechel and Ebert-Uphoff 2004). Such mechanisms have a relatively large workspace compared to their size and are generally lighter and easier to transport than serial manipulators (Roberts et al. 1998). Moreover, they have few moving parts, which give good inertial properties, high payload-weight ratio, transportability and economical

*Corresponding author. Email: ottaviano@unicas.it 
construction. These are important features for applications requiring a manipulator to be brought to work on site. Their main drawback is related to the mechanical characteristic of the cables. In fact, they can only work in tension, and this fact limits the controllable workspace (Hiller et al. 2005). Therefore, it is necessary to verify that for each end-effector configuration the cables' forces are always positive.

A number of robotic systems have been designed specifically for medical applications. Systems for aiding mobility have been proposed in Dubowsky et al. (2000), Médéric et al. (2004) and Chugo et al. (2008), for rehabilitation of limbs in Beyl et al. (2009) and Stienen et al. (2009) and cable-based manipulators have been reported for the treatment of patients for upper and lower limb rehabilitation (Mayhem et al. 2005; Castelli and Ottaviano 2009). The motivation for using robotic systems in rehabilitation is that there is strong evidence that highly repetitive movement training, with active engagement by a participant, promotes cortical re-organisation and can result in improved recovery after a stroke. Robotic devices can be used to aid automatic and repetitive training in a controlled way and to increase treatment compliance by introducing incentives to a patient, such as games or performance-related scores (Rosati et al. 2009).

In this paper, we propose a reconfigurable four-cablebased manipulator as a motion assisting and guiding system. The novelty of the approach is to reconfigure the manipulator to different types of tasks by modifying the position of end-effector attachment points and considering planar and spatial versions. Numerical simulations and experimental results are presented in the paper concerning the motion assisting system and an upper and lower limb guiding device.

\section{Design requirements for a motion assisting device}

In recent years, robotics has been successfully applied in the biomedical field. In particular, robotic rehabilitation devices can provide several advantages over traditional therapy, including objective measurements of the time course of changes in motor control of the affected limbs and semiautonomous practice of therapeutic exercises (Marlene and Cooper 1995). They can also be used to guide the movements of the patient connected to a robotic device (Mayhem et al. 2005). Often, due to economical and technological obligations, attempts have been made to adapt industrial robots to rehabilitation applications. This is not of course the best solution, as industrial and medical applications require devices with quite different requirements. Classical industrial robots have rigid structures and perform fast movements, and they are specifically designed to perform tasks without direct interaction with humans. Devices for biomedical applications rather have interaction with humans as their main purpose, so they should be safe, reliable and possibly make slow movements.
According to the nature of the application, manipulators for medical applications must also meet the requirements of (Ottaviano 2008):

- reliability, to prevent and manage failures that may affect the safety of a patient;

- compactness and portability, in order to be used in hospitals or even at home;

- simplicity, to be used by people without specific knowledge of robotics;

- flexibility, in order to meet the specific needs of each patient.

Cable-based manipulators can satisfy all these requirements thanks to their intrinsic characteristics of reliability, compactness, simplicity and re-configurability. Safety can be ensured by controlling and limiting the tension cables and performed movements.

Cable-based systems, due to their nature and characteristics, can be used for a number of applications, including those requiring a close and continuous interaction with humans, such as mobility, assistance and rehabilitation. They have a large workspace that can be exploited to treat patients for different types of therapies. Actuators are often placed on the base and the geometry of the manipulator can be varied by changing the position of the attachment points of the cables. The mechanical structure can be moved and assembled on site, for easy transport and storage, in order to even be used at the patient's home (Ottaviano 2008). The moving masses consist mainly of the cables and the mobile platform. This aspect makes the system safe and efficient in terms of energy consumption. A manipulator of this type has a relatively low cost and its maintenance is simple, important features for a system that can be used not only in hospitals but also at the patient's home. If one considers the state-of-the-art, many of the prototypes currently under study for rehabilitation have not yet been commercialised. It is therefore interesting to propose new potential applications of cable-based parallel manipulators for these applications because of their features such as ease of use, low cost, versatility, modularity and reliability.

\section{CALOWI (Cassino Low-Cost Wire Robot) prototype}

The CALOWI (Cassino Wire Low-Cost Robot) cable-based parallel manipulator has been designed and built at LARM. It possesses four cables and thus four DOF as well as a large workspace, if compared with the size of the structure. The manipulator was designed initially for fully constrained planar applications and subsequently for spatial applications as an under-constrained suspended robot. It has been tested for path-planning operations and rescue applications, for medical applications and for supporting mobility (Ottaviano et al. 2008). 
(a)

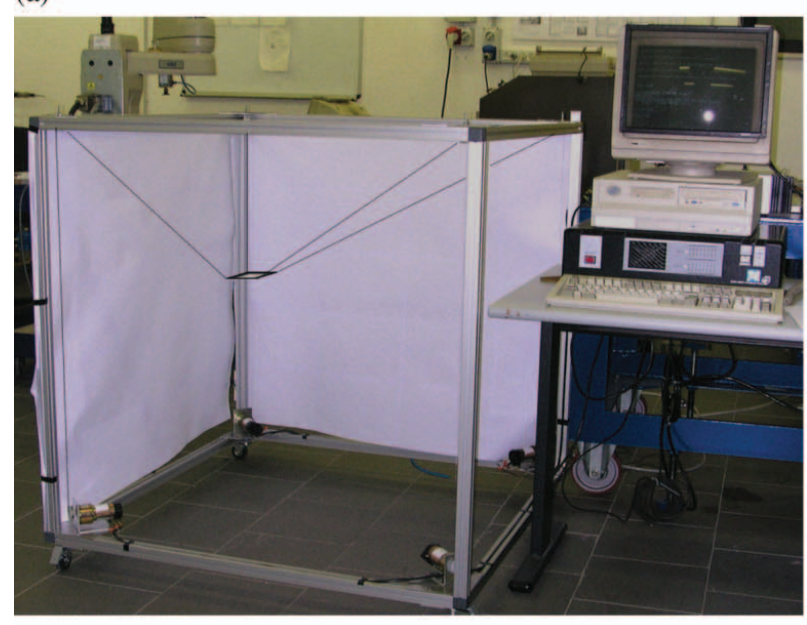

(b)

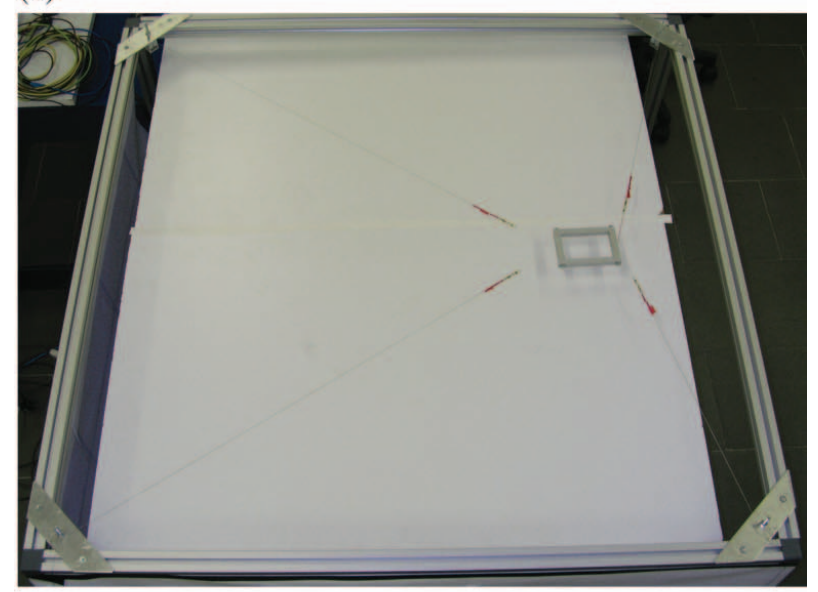

(c)

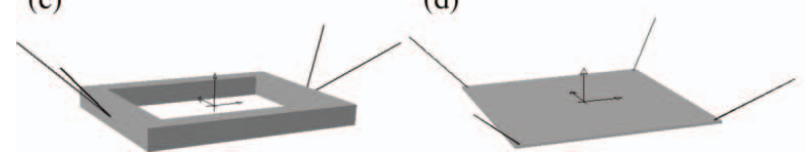

Figure 1. The CALOWI at LARM: (a) planar version, (b) spatial version, (c) 4-2 end-effector configuration, (d) 4-4 end-effector configuration.

The prototype is shown in Figure 1, the upper surface can be used for planar applications. Figure 1(b) shows the manipulator in the spatial version. The fixed structure is made of aluminium that is extremely light and yet has a good stiffness. This results in a reduced weight of the robot, which can be easily disassembled and assembled on site. The actuation system is composed of DC motors PITTMAN GM9413 DC, with a transmission ratio of 65.5:1 and maximum torque of $1.24 \mathrm{Nm}$; they are equipped with incremental encoders. Steel cables have been chosen $(E=$ $200 \mathrm{GPa}$ ) with a diameter of $0.4 \mathrm{~mm}$ and a breaking load of $70 \mathrm{~N}$. The linear density of the cable is $0.002 \mathrm{~kg} / \mathrm{m}$.

The end-effector of the manipulator can have two different configurations: the first called 4-2 with two attachment points, as shown in Figure1(c), and the second called 44 with four attachment points, as shown in Figure 1(d). The system is equipped with load sensors to monitor cable tensions (Castelli 2010).

It will be shown that this manipulator can be used for several applications as an aiding and guiding motion system.

In particular, by simply reconfiguring the manipulator, in one combination of the four possible cases shown in Figure 1, it can be used as a motion assisting device or an upper and/or lower limb aiding motion system. The planar version is used for upper limbs and the spatial version for lower limbs and as an assisting device.

\section{A system as a motion assisting device}

In the elderly, over the years, a degeneration of the nervous system and a weakening of the muscular-skeletal system can occur and, as an unfortunate consequence, this may involve the risk of falls with possible bone fractures (Winter 1990). When this happens, the first action to perform on a patient is restoring the damage (for example, through surgery) and then a rehabilitation programme must be followed regarding both physical and psychological aspects. Indeed, as a consequence of a fall, an individual will present some insecurity for the future in carrying out daily activities because he/she will always remember that event (Chugo et al. 2008). Therefore, it will be of great support as a human operator, such as a psychologist or a nurse with adequate preparation, that gives moral support to the patient, but more will be required for rehabilitation for future ambulation (Médéric et al. 2004).

A robotic system can be an alternative, but at the outset, issues related to the biomechanical gait and action of a person in the sit-to-stand transfer must be considered. In both cases there is a problem of balance of the patient, because during a walk it should always be ensured that his/her centre of gravity does not ever fall outside the feet area. Whereas during the sit-to-stand transfer, again following a possible reduced ability of the nervous system to control balance, one has to avoid front or rear rotations, called antepulsion and retropulsion, respectively, as shown in Figure 2, which may lead to possible falls of the individual (Castelli and Ottaviano 2009). The proposed application consists of a robotic system based on a four-cable-suspended manipulator, which can be used to help people with motion disabilities in lower limbs. In particular, the sit-to-stand transfer operation is analysed.

In addition to the choice of the manipulator, for this application, it is important to consider an appropriate sling system, as it represents the connection/interaction between the robotic system and a patient.

The sling must be designed to sustain the body in a stable and secure way. It must not cause problems to the shoulders and, at the same time, leave the legs and hip 


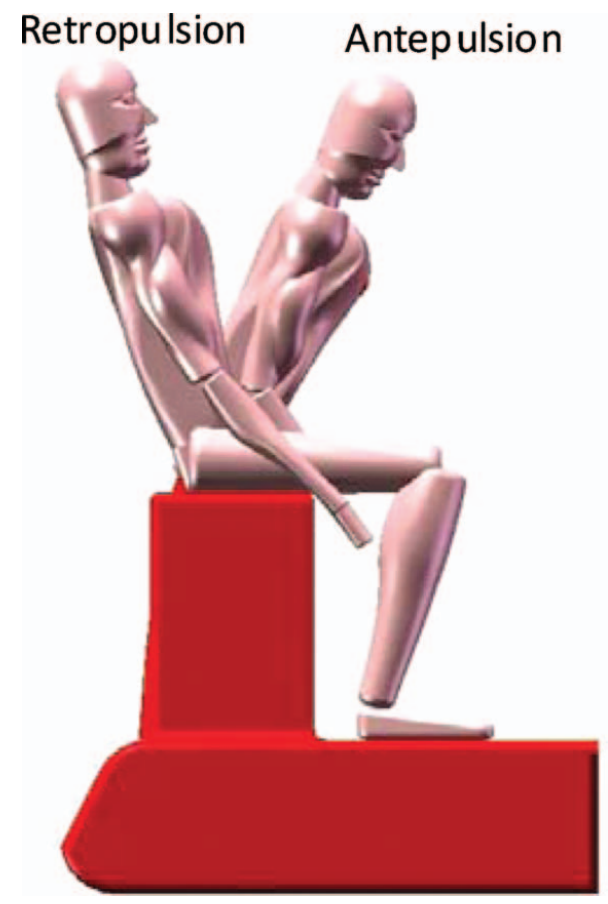

Figure 2. Schemes for antepulsion and retropulsion for a seated individual.

articulation free to move. It should also be useful in countering antepulsion and retropulsion.

Sling systems currently available in the market are commonly used for skydiving or as safety systems for workers (http://www.liko.com). They are generally developed for patients who have difficulty in maintaining balance and have low muscle tone in their legs. The sling transmits most of the load to the groin and is therefore very suitable for patients particularly sensitive to pressure on the upper parts of the body. There is also a special padded crotch which relieves the pressure.

A further advantage is the suspension brackets which do not impede the natural movement of the upper limbs during walking, but cannot be used by people with spinal problems.

The systems available in the market also have the disadvantage of not being easily removable and worn by a person with reduced mobility or in the seated position. The optimum solution should be a sort of safety-vest, easy to wear, that allows freedom from back and groin strains. Furthermore, the cables' attachment points should be placed above the centre of gravity of the individual to prevent antepulsion and retropulsion.

Main characteristics that the robotic system must meet can be deduced from the requirements of the operation to be performed, which consists of the sit-to-stand transfer.

As an example, for an individual with a height of $1.90 \mathrm{~m}$ and a mass of $150 \mathrm{~kg}$, which is the 95th percentile of the size of an adult (DINBelg website 2010), steel cables with a diameter of $2 \mathrm{~mm}$ may be chosen. Each cable can support $337 \mathrm{~kg}$ with a breaking load equal to $1570 \mathrm{~N} / \mathrm{mm}^{2}$. Depending on the cable, the diameter of the pulleys to be used should be $135 \mathrm{~mm}$, while the actuation system must have a torque of $230 \mathrm{Nm}$ for each cable. Given the limit tension and low speed, i.e. of $0.20 \mathrm{rad} / \mathrm{s}$ for security reasons, a power of $50 \mathrm{~W}$ is required for each motor (Castelli and Ottaviano 2009).

\subsection{Modelling of the system}

On the basis of the above-mentioned design considerations, a model in the ADAMS environment has been developed to analyse the dynamic behaviour and feasibility of the application. The model consists of the cable-based robotic system CALOWI and a model of the human body, both used to test and simulate the interaction between the operation support system and the human body and the whole application.

\subsubsection{A human body model}

Modelling and simulation of the human body is a complex task and requires extensive studies, simulations and experimental verifications (Marlene and Cooper 1995). In this context, a simplified model has been developed. An in-depth analysis of the biomechanics of the human body and the complex actions that it perceives and experiences is beyond the scope of this work, and they may be regarded as specific future developments. The human body model has been chosen as the best compromise between computational complexity and requested capabilities for simulating the motion. It is based on a simplified 3D model of the human body that consists of 11 segments, which are connected by 10 revolute joints (Castelli 2010). Each segment is modelled by a relatively simple geometry that allows full body symmetry with respect to the sagittal plane (leftright symmetry), as shown in Figure 3. Segment masses and dimensions in Table 1 have been assumed according to anthropometric data reported at http://www.dinbelg.be/ anthropometry.htm.

Each segment has been modelled with a uniform density and centre of mass to be coincident with the centre of volume. The proposed human model has been validated

Table 1. Main data of the CAD model for the human body in Figure 3.

\begin{tabular}{lcc}
\hline Body segments & Dimension $\left(\mathrm{mm}^{3}\right)$ & Mass $(\mathrm{kg})$ \\
\hline Head + neck + torso + pelvis & $450 \times 890 \times 235$ & 61 \\
Upper arm & $110 \times 340 \times 110$ & 3 \\
Lower arm & $80 \times 300 \times 46$ & 2.5 \\
Upper leg & $23 \times 460 \times 23$ & 8.5 \\
Lower leg & $126 \times 440 \times 126$ & 6 \\
Foot & $131 \times 250 \times 60$ & 1.5 \\
Human body & $670 \times 1857 \times 315$ & 104 \\
\hline
\end{tabular}




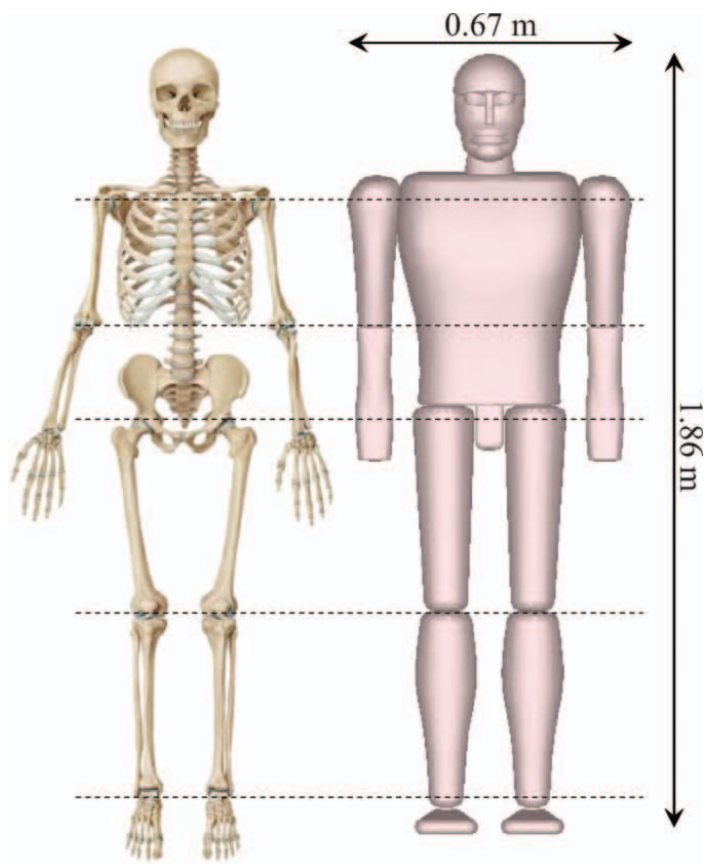

(a)

\section{Total body mass $104 \mathrm{~kg}$}

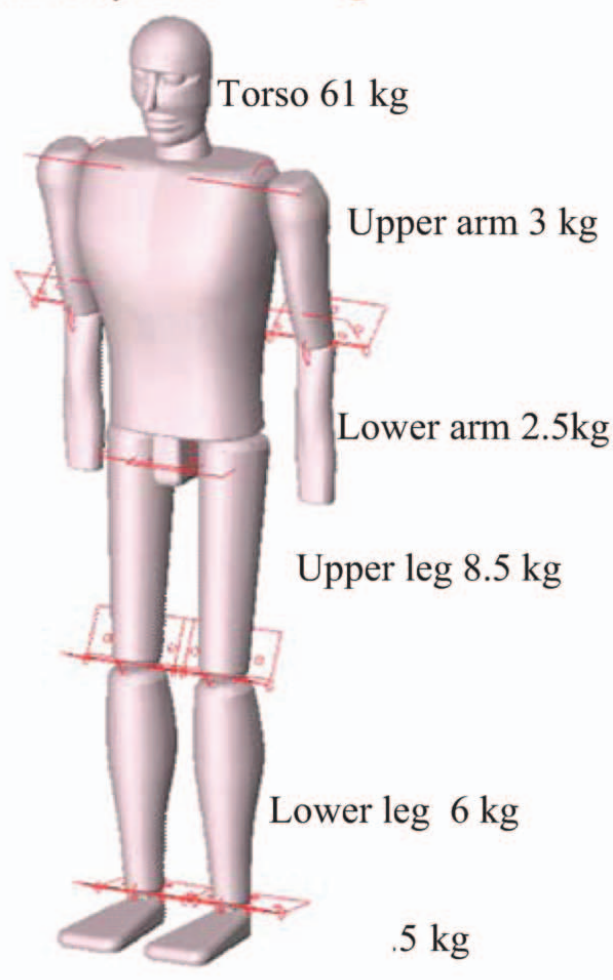

(b)

Figure 3. Scheme of a 3D model of human body: (a) comparison with a realistic scheme and (b) model with related parts and masses. through several simulations and experimental data are reported in Castelli (2010).

\subsubsection{A model for the cable-based parallel manipulator and assisting application}

The manipulator's structure has dimensions of $2300 \times 2700$ $\times 2300 \mathrm{~mm}$ and was modelled as a rigid body. The endeffector has also been modelled as a rigid body, of 300 $\times 105 \times 216 \mathrm{~mm}$ and a mass of $3 \mathrm{~kg}$. The chosen cable for the simulation is made of steel with a diameter of 2 $\mathrm{mm}$, a Young's modulus of $200 \mathrm{GPa}$, a breaking load of $3300 \mathrm{~N}$ and a mass of $0.02 \mathrm{~kg} / \mathrm{m}$.

Cables have been modelled as mass-less linear springs with an elastic coefficient $k$. According to the results shown in Castelli (2010), the manipulator cables' masses were not considered in modelling because their effect on the endeffector positioning is negligible. Indeed, in this case, the ratio between the end-effector mass (sling and human body) and the total mass of the cables is equal to 200 .

Therefore, it has been verified numerically and experimentally that the resulting position error is negligible for the case under study (Castelli and Ottaviano 2009).

The interaction between the model of the human body and the robotic system is achieved through a sling attached to the upper torso and four cables connecting the sling to the fixed frame of the manipulator, as shown in Figure 4.

In the proposed model the reference system is considered to be fixed on the lower surface of the structure.

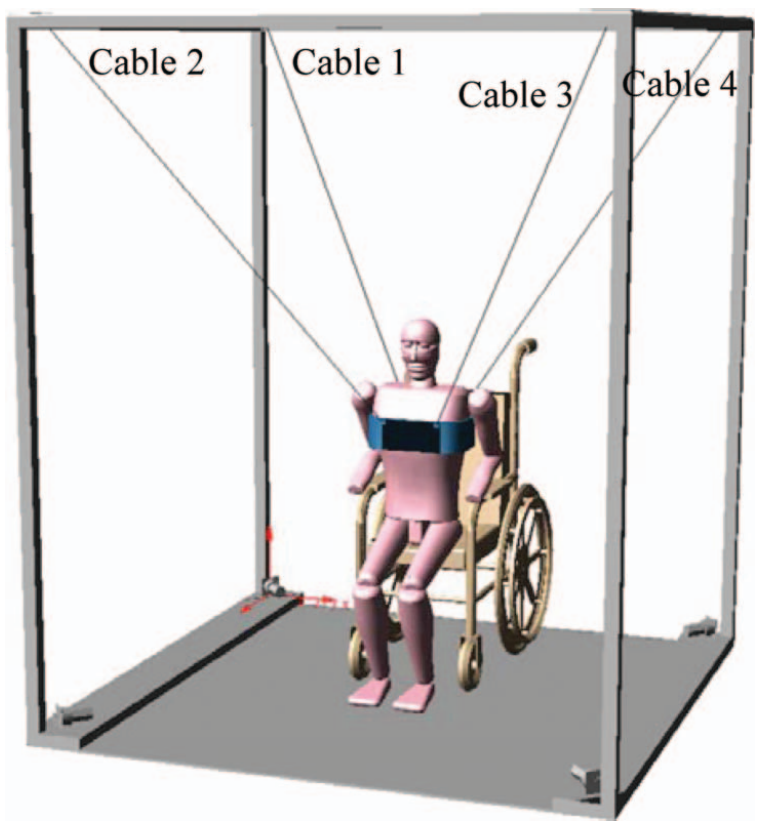

Figure 4. A model of the human body and cable-based manipulator. 


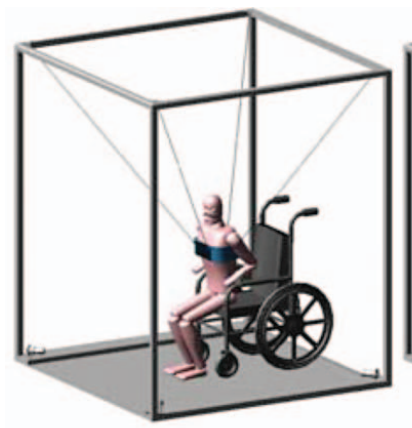

(a)

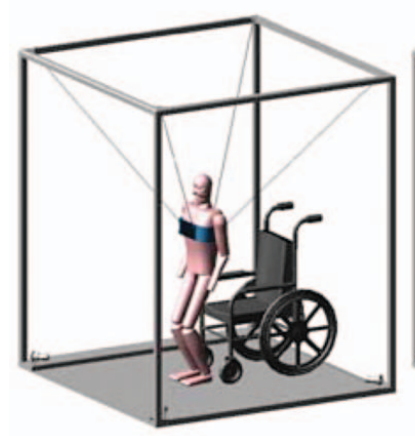

(c)

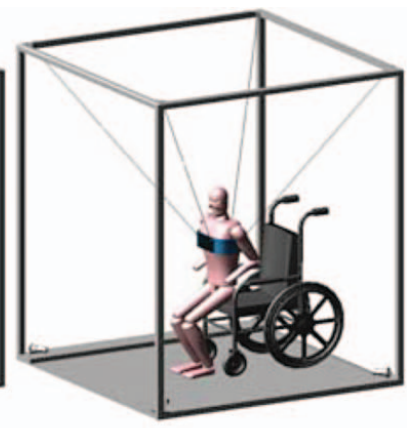

(b)

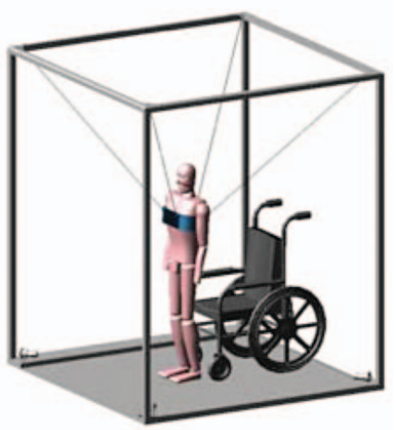

(d)
Figure 5. A sequence (a-d) of the standing operation at times 1, 4,7 and $10 \mathrm{~s}$, respectively.

The model of the human body interacts with the ground through the feet and with the wheelchair through the pelvis. For dynamic simulations such contacts have been modelled assuming a suitable friction coefficient.

Several simulations were carried out according to the model in Figure 4.

In particular, Figure 5 shows a simulation of the action of the sit-to-stand transfer. The duration of the simulation is $11 \mathrm{~s}$ : during the first second the system is left free to reach the static equilibrium, then the manipulator lifts the patient until the standing configuration is reached.

It is worth noting that the human body does not exert any force but acts as a dead weight, and the arms do not interact with the wheelchair during the movement. Figure 5 shows the motion sequence during the simulation.

Figure 6(a) shows the cables' variation for the lifting operation, Figure 6(b) shows the cables' tensions, and the angular velocity of the motors is shown in Figure 6(c).

Note that lengths and forces of cables 1 and 4, like those of cables 2 and 3, are not exactly equal because the human body model is not perfectly centred with respect to the frame.

In Figure 6(d) the required power is shown, and values are below the designed ones. Figure 6(e) shows the centre of mass position of the human body model, and Figure 6(f) shows the orientation of the body with respect to the $Y$ axis.
It is important, for people with disability in lower limbs, to study reactions of the feet to the ground and those in the knee joints. Figures $7(a, b, c)$ show results for the contact forces along the $Z$ axis of the foot, knee and body.

It is worth noting that using this system, the cables support $90 \%$ of the body weight, which may be interesting for people with reduced strength in the legs.

\subsubsection{Experimental tests of cable-based parallel manip- ulator and assisting application}

Laboratory tests were carried out with a prototype shown in Figure 8(a). The tests were performed with the prototype of the CALOWI manipulator in Figure 1 used in the 4-4 configuration for the end-effector. During the test, with a duration of $11 \mathrm{~s}$, tensions in the cables were acquired through the system based on the load sensors.

Figure 8(b) shows experimental results obtained during the test. Note that the numerical and experimental results cannot be directly compared because they refer to manipulators with different sizes, loads and types of cables. Nevertheless, the qualitative comparison shows a good match.

\subsubsection{A system for a motion assisted upper limb movements}

Rehabilitation is a discipline which deals with health problems related not only to age but also to consequences of trauma to the limbs, spine, or nervous system. Medical rehabilitation is often referred to as physical therapy (PT), occupational therapy (OT) and psychological support. For PT and OT, primarily therapists and medical staff are engaged and the treatment is often based on an exercise with close supervision in order to assist the patient in functional recovery. Unfortunately, this type of treatment is limited in time due to shortage of medical personnel and appropriate facilities. Robotic systems can support medical and physiotherapy activities, with their safety features, reliability, customisation, repeatability and ability to vary the forces and movements. Currently, few robotic systems are dedicated to the rehabilitation of the upper limbs (e.g. Kiguchi and Fukuda 2004; Sugar et al. 2007).

To define the actions that a robotic system has to perform on a human arm, it is important to correctly model both the arm and the body. The upper limb is composed of three parts: arm, forearm and hand. The skeleton consists of the humerus, which is articulated through the elbow with the two bones of the forearm, the radius and ulna. The hand articulates with the forearm through the wrist, whose skeleton is a system of eight carpal bones. The skeleton of the hand is composed of metacarpal bones (metacarpus). The skeleton of the fingers of the hand is composed of three bones called phalanges. One exception is the thumb, which is formed by only two phalanges (Gray 1918). The number 


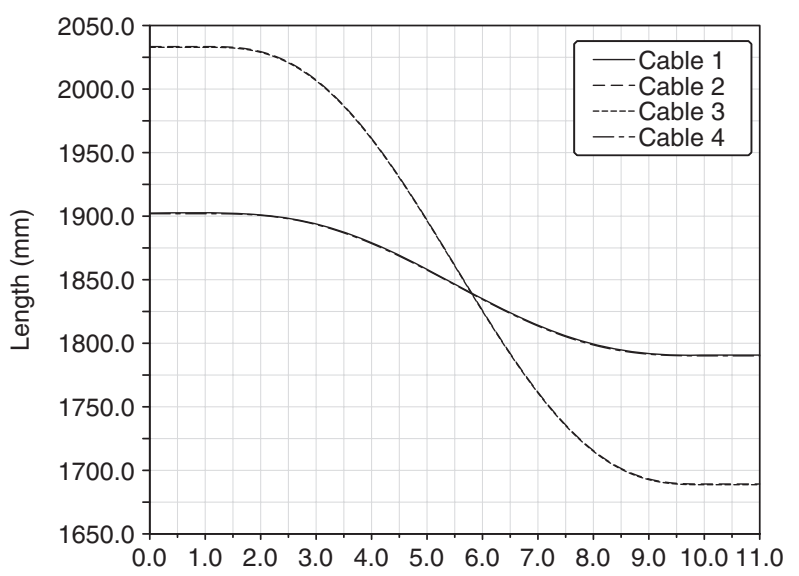

(a)

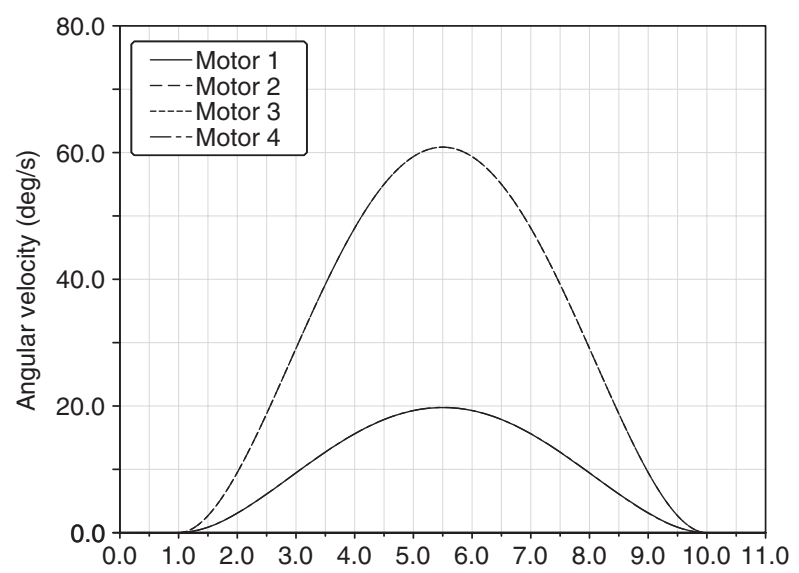

(c)

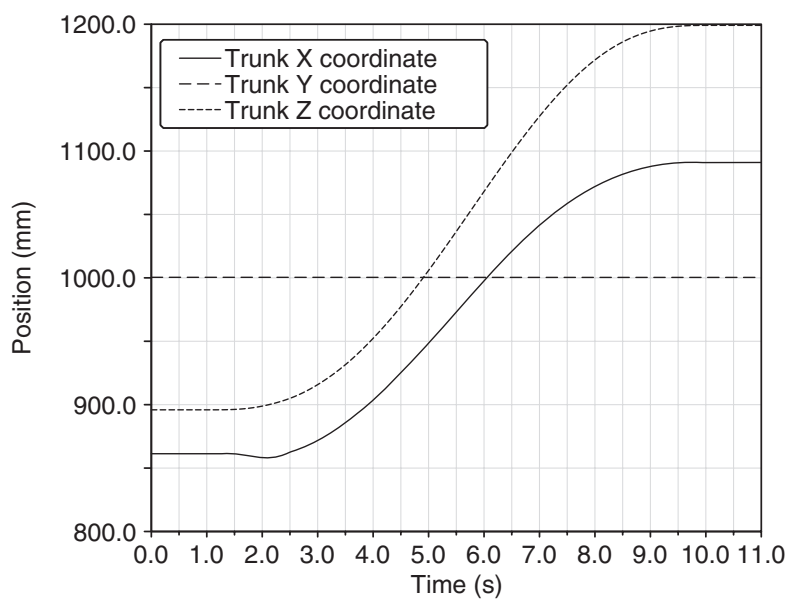

(e)

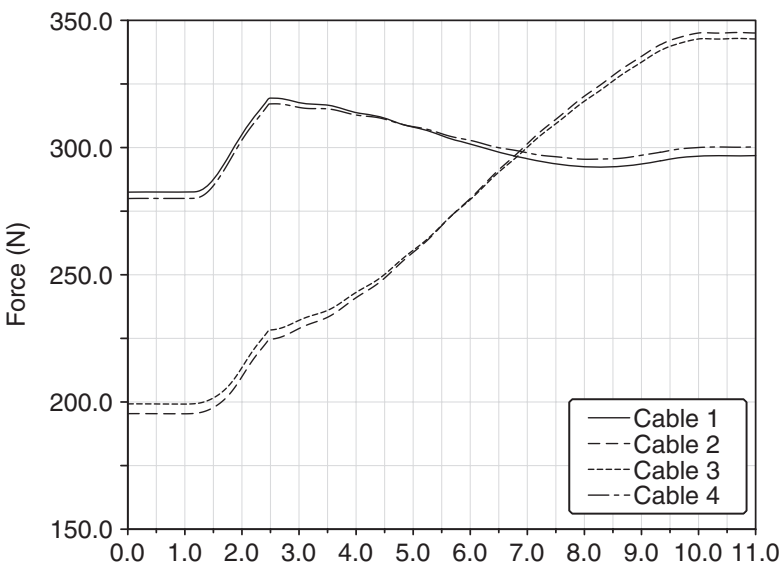

(b)

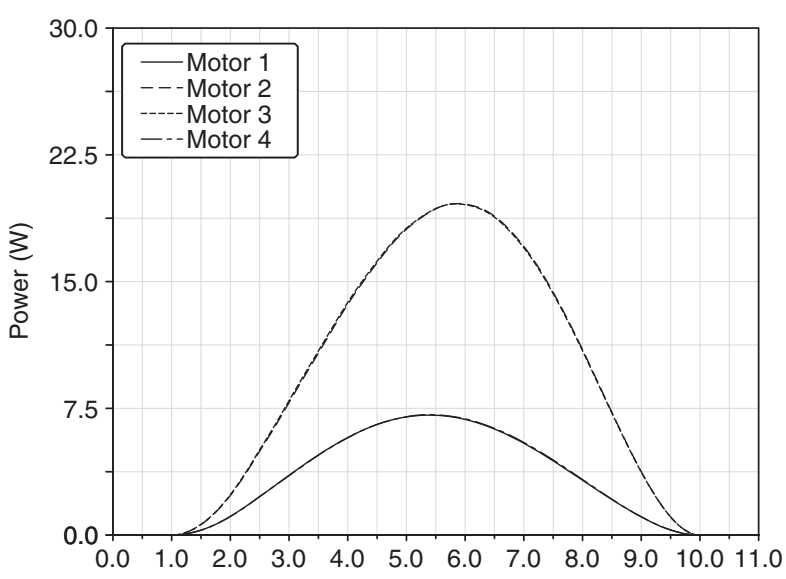

(d)

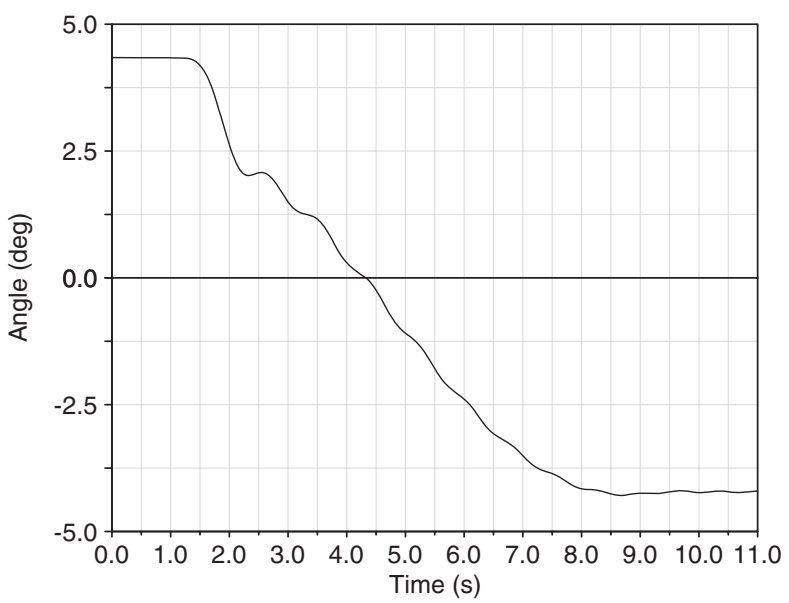

(f)

Figure 6. Numerical results for the simulation in Figure 5 (duration $11 \mathrm{~s}$ ): (a) cables' lengths, (b) cables' tensions, (c) angular velocity of the motors, (d) motors' power, (e) centre of mass position, (f) orientation of the human body with respect to the $Y$ axis. 


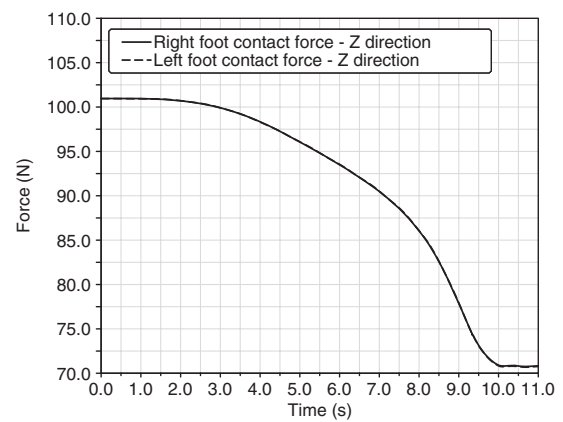

(a)

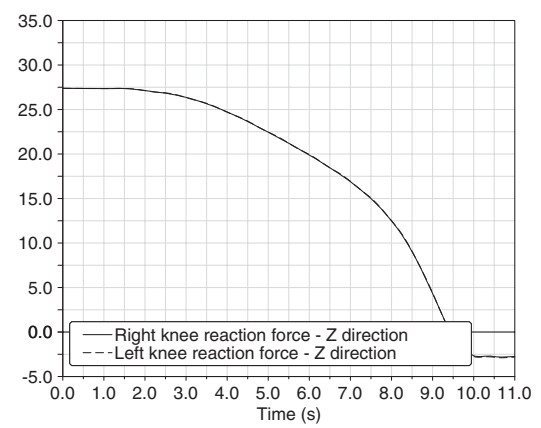

(b)

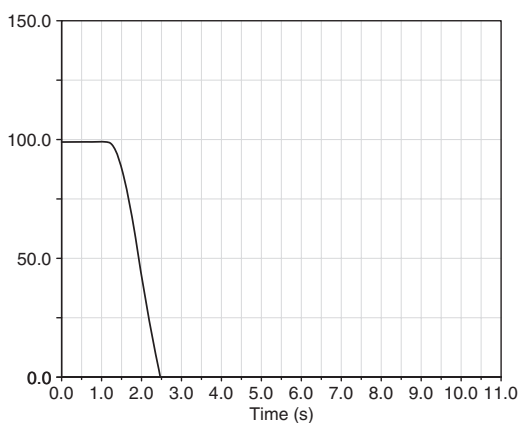

(c)

Figure 7. Numerical results for the simulation in Figure 5 (duration $11 \mathrm{~s}$ ): (a) reaction forces at feet, (b) reaction forces at knees, (c) reaction force at the wheelchair.

of bones, and therefore the number of joints, increases towards the fingers. For modelling the upper limb, the most important joints are the shoulder, elbow, wrist and joints of the hand. In this context, the objective is to evaluate the characteristics of the CALOWI manipulator as a motion aiding system of the upper limb. The motions of the wrist and hand have not been actively implemented; therefore the joints considered are the shoulder and the elbow.

\subsection{An upper limb model}

According to the considerations reported in Castelli (2010), a model of the upper limb has been developed in the ADAMS environment, whose parameters are reported in

Table 2. A model and parameters of the upper limb.

\begin{tabular}{lcc}
\hline & Upper arm & Lower arm + hand \\
\hline Dimensions $\left(\mathrm{mm}^{3}\right)$ & $110 \times 110 \times 340$ & $80 \times 60 \times 300$ \\
Mass $(\mathrm{kg})$ & 3 & 2.5 \\
CAD model & & \\
\hline
\end{tabular}

Table 3. A description of the joints' model.

\begin{tabular}{lrlll}
\hline \multirow{2}{*}{ Joint } & $\begin{array}{r}\text { Position } \\
(\mathrm{mm})\end{array}$ & $\begin{array}{c}\text { Type of } \\
\text { joint }\end{array}$ & $\begin{array}{c}\text { Rotation } \\
\text { axis }\end{array}$ & $\begin{array}{c}\text { ADAMS } \\
\text { model }\end{array}$ \\
\hline Shoulder & 510.0 & Spherical & - & \\
& -50.0 & & & \\
& -160.0 & & & \\
584.6 & Revolute & $Y$ & \\
Elbow & -50.0 & & & \\
& 147.0 & & & \\
Grasping for the hand & 559.6 & Revolute & $Y$ & \\
& -50.0 & & & \\
End-effector & 418.0 & & \\
& 150.0 & Planar & $Y$ & \\
& 0.0 & & \\
\hline
\end{tabular}

Tables 2 and 3, together with the torso of the human body, to simulate a number of movements.

It should be noted that the size and shapes for the model of the upper limb are the same as those used to develop the

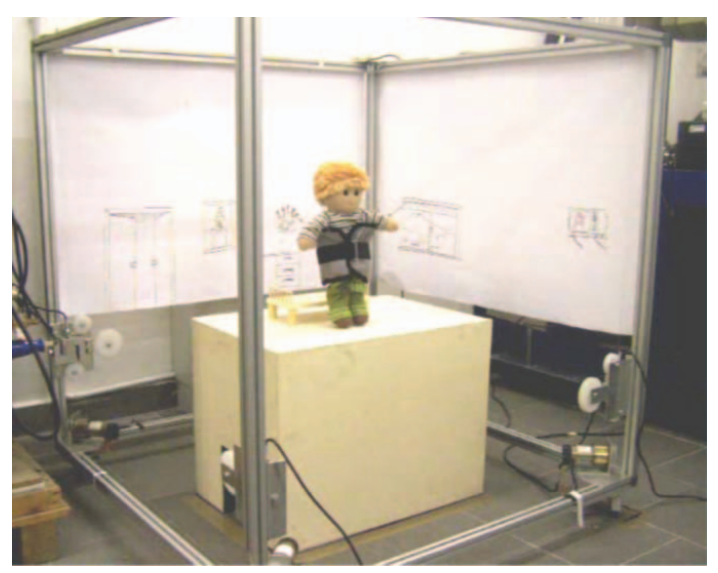

(a)

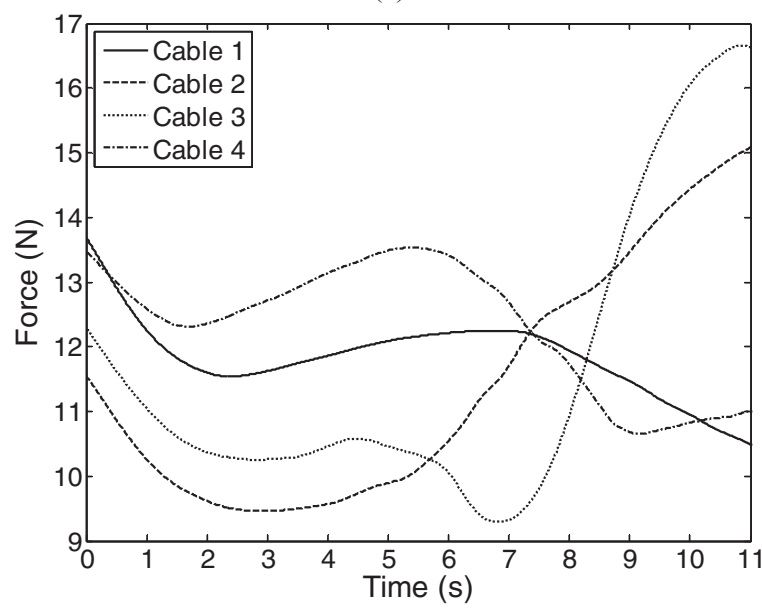

(b)

Figure 8. Experimental tests for the standing operation: (a) layout and (b) results for the cables' tensions. 


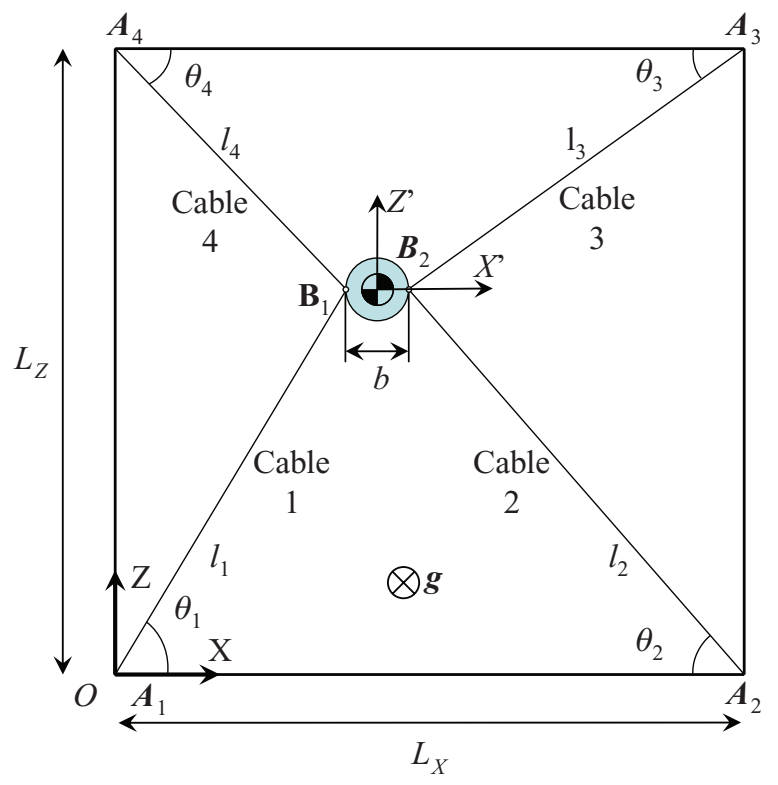

Figure 9. A scheme and kinematic parameters for a planar 4-2 cable manipulator.

model of the human body described in the previous section. In this case, a spherical joint has been used for simulating the shoulder and one revolute joint for the elbow, as reported in Table 3.

\subsection{A model for the cable-based parallel manipulator and assisting motion application}

A model of the cable-based manipulator was developed in the ADAMS environment (Castelli 2010). Even in this case it refers to the CALOWI manipulator, but in the planar version with an end-effector configuration 4-2, as shown in the scheme of Figure 9.

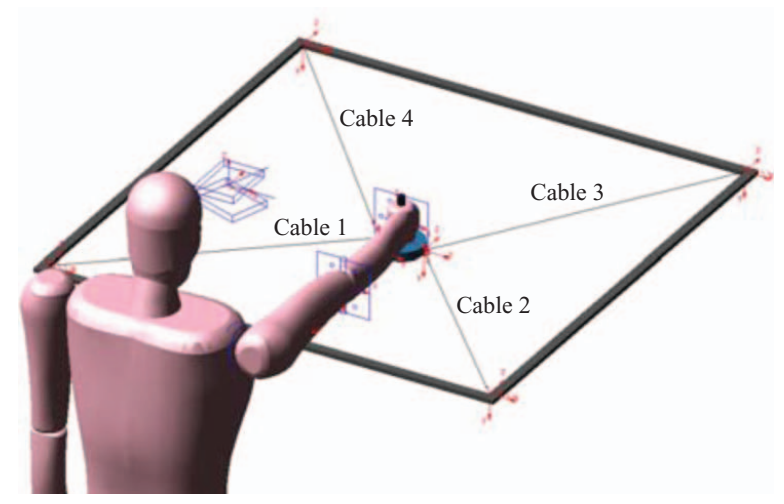

Figure 10. A model in the ADAMS environment of the system for the motion aiding of upper limbs.
In Table 4 data are reported for the model of the manipulator in Figure 9. For the present purposes, an ad hoc rigid body has been considered as the end-effector. It is composed of a cylindrical element for grasping and a disk-shaped element in order to slide on a flat surface and to allow some trajectories that can be set. In Figure 10 the overall model of the system is reported, consisting of the planar cable manipulator and the human body model.

The model of the manipulator was developed according to the parameters given in Table 4. The fixed base and end-effector were modelled as rigid bodies. Elasticity of the cables was considered, while the mass was neglected. The cables' characteristics are the same as those for the manipulator described in the previous section $(E=200$ $\mathrm{GPa}, \varnothing=0.4 \mathrm{~mm}, \rho=0.002 \mathrm{~kg} / \mathrm{m}, F_{\max }=70 \mathrm{~N}$ ).

The motion of the end-effector has been constrained in the $X Z$ plane and, therefore, gravity does not affect the system. The task of this system is to assist or guide the arm motion of people with impaired mobility to the upper limbs.

As previously stated, this system can be thought of as a useful support to traditional therapies of rehabilitation.

The steps followed in rehabilitation therapy are mainly the analysis of a patient's status, including anthropometric data, the action of planning and performing exercises with the aid of a physiotherapist.

A robotic system can be used in the last two stages, i.e. it can be complementary in defining the tasks and movements, through appropriate simulation, and can actively aid in motions in the process of rehabilitation itself.

Simple trajectories can be chosen, such as a circle or a square. The area involved in the movement must be determined depending on the position of the person with respect to the robotic system and anthropometric data, such as the size of the limb. In the scheme of Figure 11 a top view of the manipulator is shown, in which the centre of the shoulder joint has been denoted by $C_{b}$. By placing the individual in front of the manipulator, as in the ADAMS model in Figure 10 , the workspace boundary of the upper limb can be approximated by a circle of radius $R_{b}$ with $C_{b}$ as the centre, assuming that the torso of the patient is fixed as the fixed base.

According to the parameters reported in Table 5, trajectories can be planned for a movement from the point marked by $S$, which describes a circle as shown in Figure 11(a) or a square as shown in Figure 11(b).

Depending on the location and size of the geometric objects described in Table 5, through the inverse kinematics of the manipulator (Castelli 2010), the variation of the cables' lengths can be derived to let the end-effector, which is the coupling device between the manipulator and the hand, follow the desired trajectory.

A simulation for the variation of cables' lengths for a circular motion is shown in Figure 12(a), while that for 
Table 4. Parameters of the planar four-cable manipulator in Figure 9.

\begin{tabular}{|c|c|c|c|c|c|c|c|}
\hline \multirow[t]{3}{*}{$L_{x}(\mathrm{~mm})$} & \multirow{3}{*}{$\begin{array}{c}L_{z}(\mathrm{~mm}) \\
1000 \\
\mathrm{~A}_{1}\end{array}$} & \multirow{3}{*}{$\begin{array}{c}b(\mathrm{~mm}) \\
100 \\
\mathrm{~A}_{2}\end{array}$} & \multicolumn{3}{|c|}{ end-effector mass (kg) } & \multicolumn{2}{|c|}{ Gravity direction } \\
\hline & & & & 0.5 & & \multicolumn{2}{|c|}{$Y^{+}$} \\
\hline & & & $\mathrm{A}_{3}$ & $\mathrm{~A}_{4}$ & & $\mathrm{~B}_{1}$ & $\mathrm{~B}_{2}$ \\
\hline$X(\mathrm{~mm})$ & 0 & 1000 & 1000 & 0 & $X^{\prime}(\mathrm{mm})$ & -50 & 50 \\
\hline$Z(\mathrm{~mm})$ & 0 & 0 & 1000 & 1000 & $Z^{\prime}(\mathrm{mm})$ & 0 & 0 \\
\hline
\end{tabular}

the square is shown in Figure 12(b). These values serve as an input for the experimental tests carried out with the cable-based manipulator prototype.

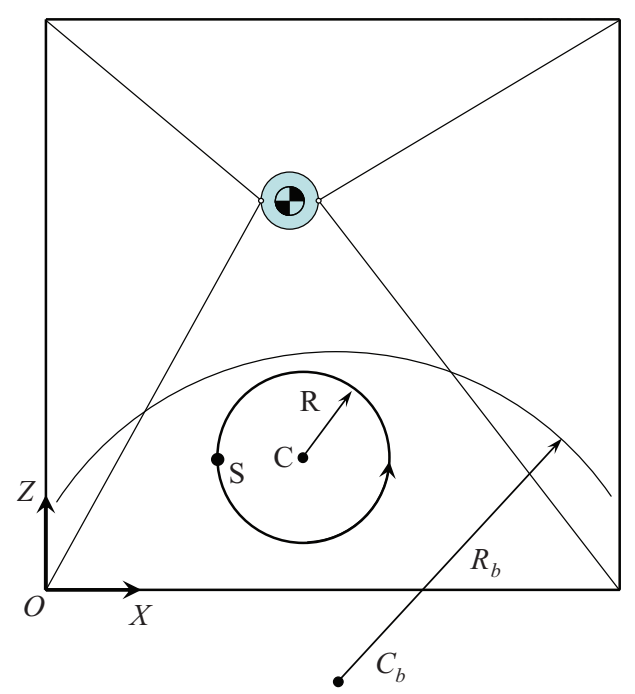

(a)

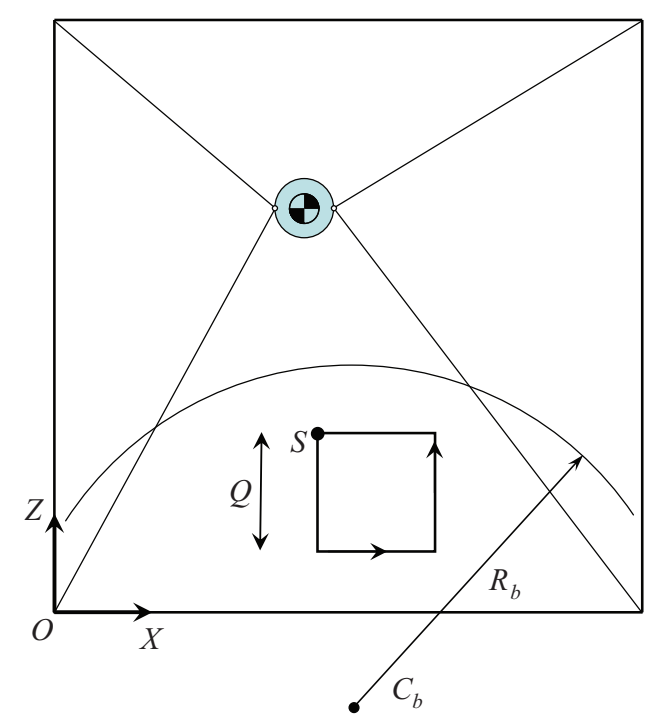

(b)

Figure 11. A scheme of the proposed layout for an aiding motion system: (a) circular trajectory and (b) squared trajectory.

\subsection{Experimental tests for the cable-based parallel manipulator and assisting motion application}

Depending on the proposed tasks, experimental tests were carried out by using the prototype CALOWI in the planar version and the end-effector configuration 4-2, as shown in Figure1.

The layout for experimental tests is shown in Figure 13(a), while Figure 13(b) shows the end-effector during the manipulation, which is reported in Figure 11(b).

The variation of the cables' lengths obtained during the simulation has been implemented in the robot controller for the execution of the proposed task. The experimental system, based on load cells as sensors, has been used to experimentally evaluate the forces acting on the cables.

Figures 14 and 15 show a comparison between the forces measured experimentally and those obtained from simulations in the ADAMS environment.

It is worth noting that the reported results are preliminary, since they have been obtained by a movement of the manipulator's end-effector without interaction with the upper limb of a person.

The comparison shows a good match from a qualitative and quantitative point of view, which encourages the continuation of the application considering tests on subjects with suitable protocols and medical personnel.

\subsection{A system for motion assisted lower limb movements}

For the implementation of a robotic system in assisting medical personnel for physiotherapy and rehabilitation of the lower limbs, it is essential to correctly model the human body and particularly the lower limbs. The lower limb of the human body is composed of three parts: thigh, leg and foot. The skeleton is formed by the femur of the thigh (femur), which through the knee is articulated with the two bones of the leg, the tibia and fibula. The knee joint is also part of a

Table 5. Parameters for trajectories in Figure 10.

\begin{tabular}{lcccc}
\hline \multicolumn{3}{c}{ Circumference } & \multicolumn{2}{c}{ Square } \\
\hline$S(\mathrm{~mm}, \mathrm{~mm})$ & $C(\mathrm{~mm}, \mathrm{~mm})$ & $R(\mathrm{~mm})$ & $S(\mathrm{~mm}, \mathrm{~mm})$ & $Q(\mathrm{~mm})$ \\
300,250 & 450,250 & 150 & 450,300 & 200 \\
\hline
\end{tabular}




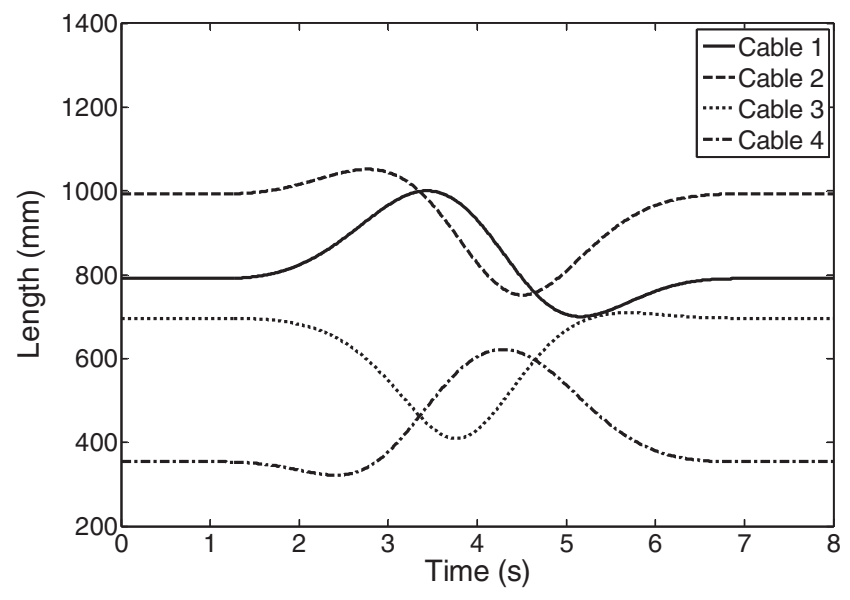

(a)

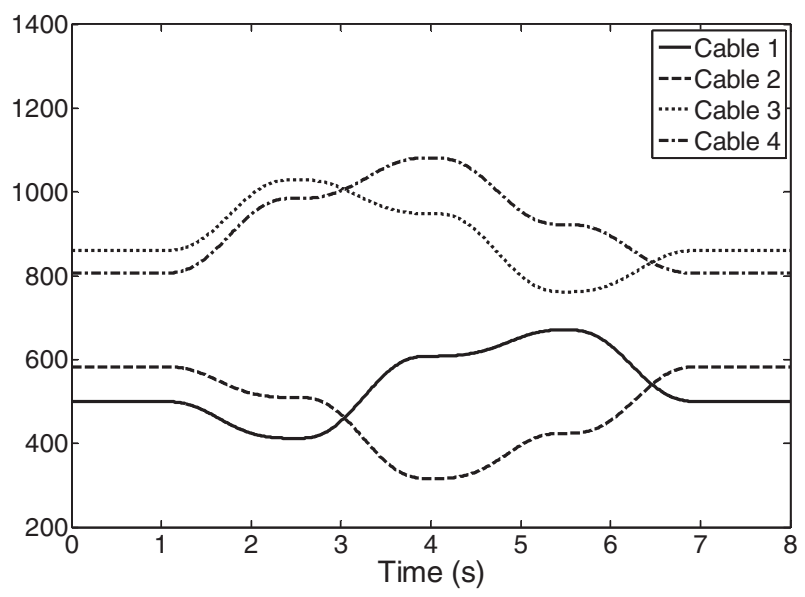

(b)

Figure 12. Variations of the cables' lengths for the proposed trajectories: (a) circumference and (b) square.

disk-shaped bone, the patella. The foot is articulated in the leg by the ankle, whose skeleton is a system of eight short bones called tarsus. The skeleton of the foot is composed of metatarsal bones (metatarsus). The skeleton of the toes is composed of three bones, called phalanges. One exception is the big toe that is formed by only two phalanges (Gray 1918). In analogy with the upper limb, the number of bones and joints increases towards the phalanges.

For modelling the lower limb, the most important joints are the hip, knee, ankle, and joints of the foot.

\subsubsection{A lower limb model}

The objective of this task is to evaluate the characteristics of the CALOWI manipulator as an assisting motion system for the thigh and leg. Therefore, in the following, the foot and the articulation of the ankle have not been actively implemented, while the hip and knee joints are considered.

The hip joint can be modelled by a ball joint, with characteristics very similar to the articulation of the shoulder.

Therefore, the leg can rotate about the left and right sagittal axis (abduction and adduction), about the front and rear transverse axis (flexion and extension) and about the median axis (vertical) (Gray 1918).

The knee joint can be modelled by a revolute joint, with characteristics very similar to the articulation of the elbow.

Therefore, the mobility of the lower leg with respect to the thigh is related only to the rotation about the transverse axis.

The developed ADAMS model of the human body and legs is used here to simulate some lower limb movements.

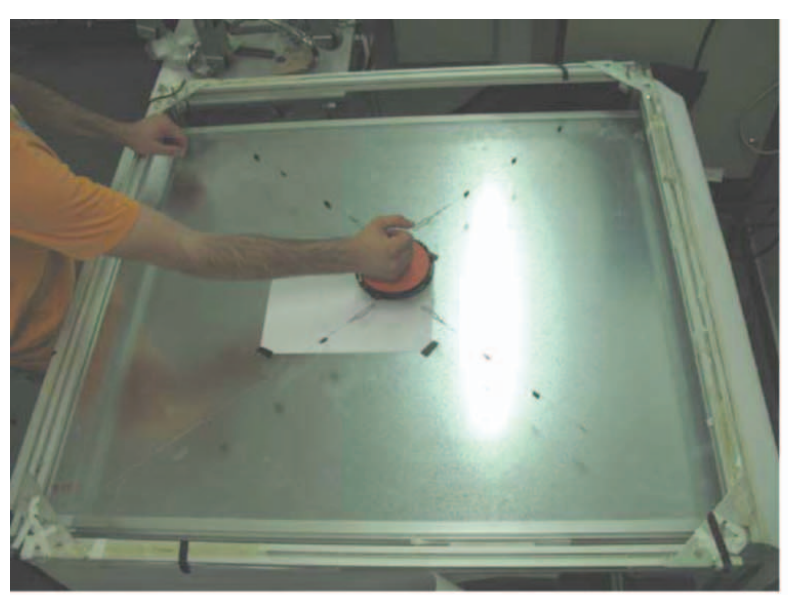

(a)

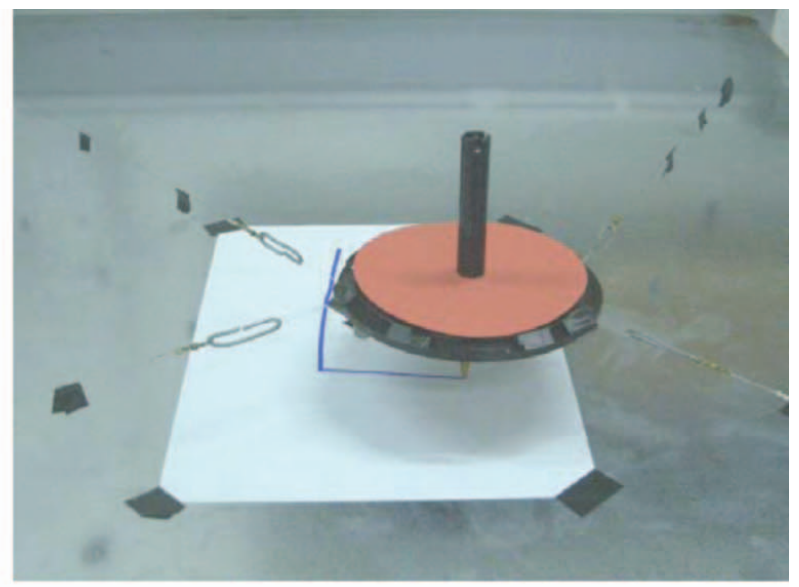

(b)

Figure 13. CALOWI prototype used as assisting motion device for upper limbs: (a laboratory layout; b) tests for trajectory in Fig.11b). 


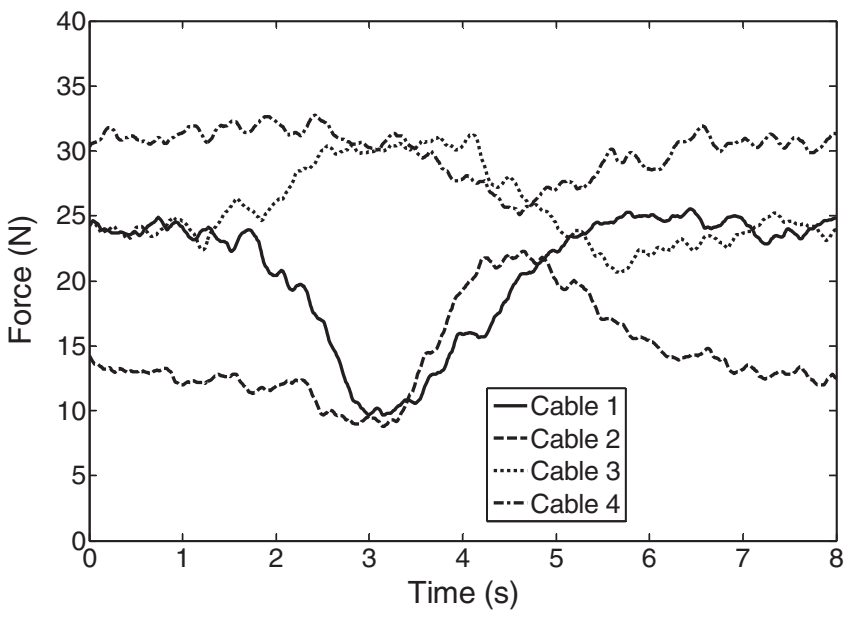

(a)

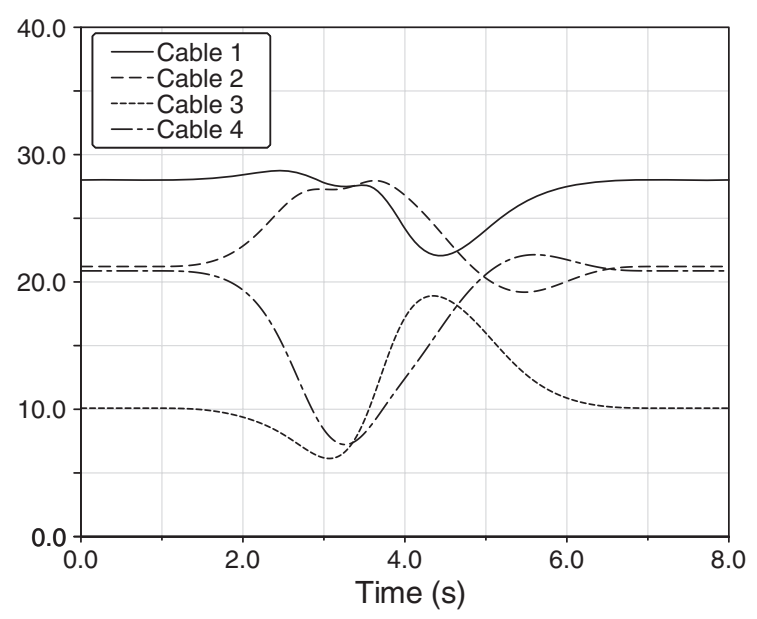

(b)

Figure 14. Forces acting in the cables during the movement in Figure 11(a) for a circular trajectory: (a) experimental test and (b) numerical simulation.

Each part of the model of the human body was modelled as a rigid body. In this case, given the type of movements, joints of the limb were modelled using a spherical joint for the hip and one revolute for the knee.

\subsubsection{A model for the cable-based parallel manipulator and assisting motion application}

The model of the human body in Figure 16 is seated on a wheelchair, which is considered attached to the fixed frame of the manipulator.

The only moving parts are those inherent to the right lower limb: thigh, leg and right foot (Castelli et al. 2008).

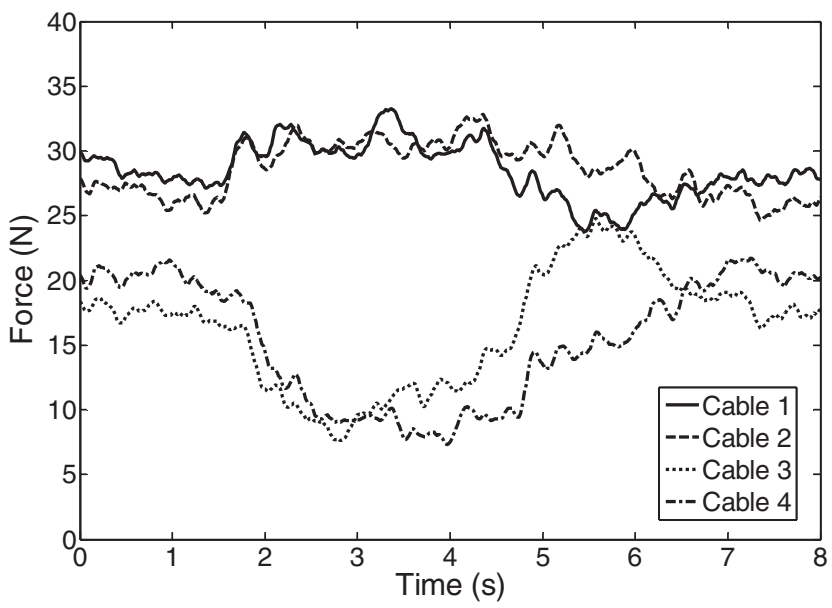

Figure 16 shows the model of the CALOWI manipulator that interacts with the human body, simulating the aiding motion application.

The model of the prototype was built according to the parameters given in Table 6, in agreement with the dimension of the built prototype. The fixed base and end-effector were modelled as rigid bodies. For this simulation, the elasticity of the cables was taken into account, while the mass of cables was neglected.

The cable characteristics are the same as those of the prototype $(E=200 \mathrm{GPa}, \varnothing=0.4 \mathrm{~mm}, \rho=0.002 \mathrm{~kg} / \mathrm{m}$, $\left.F_{\text {max }}=70 \mathrm{~N}\right)$.

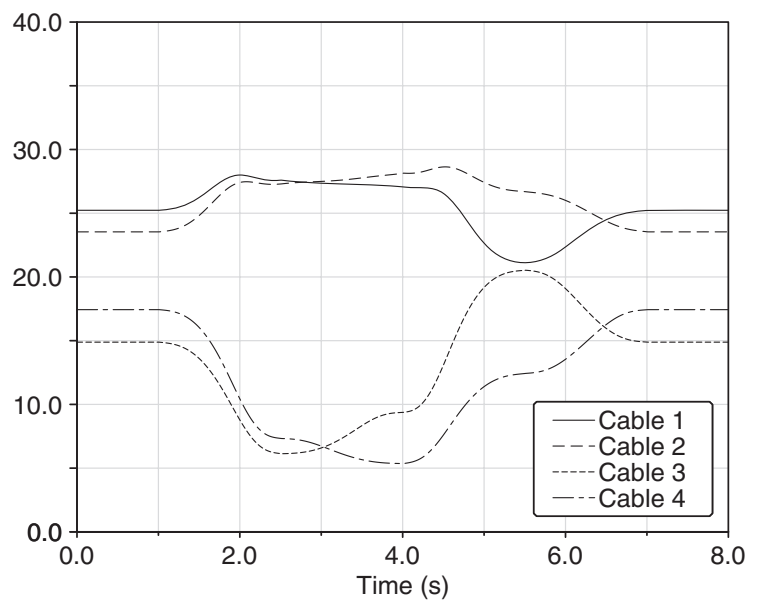

(b)

Figure 15. Forces acting in the cables during the movement in Figure 11(b) for a squared trajectory: (a) experimental test and (b) numerical simulation. 
Table 6. Parameters of the spatial manipulator in Figure 16.

\begin{tabular}{lcccccrrr}
\hline$L_{X}(\mathrm{~mm})$ & $L_{Y}(\mathrm{~mm})$ & $L_{Z}(\mathrm{~mm})$ & $b_{X}(\mathrm{~mm})$ & $b_{Y}(\mathrm{~mm})$ & $h(\mathrm{~mm})$ & End-effector mass $(\mathrm{kg})$ & Gravity direction \\
\hline 1000 & 1000 & 1000 & 100 & 200 & 10 & & 0.3 & $Z^{\mathrm{t}}$ \\
\hline & $\mathrm{A}_{1}$ & $\mathrm{~A}_{2}$ & $\mathrm{~A}_{3}$ & $\mathrm{~A}_{4}$ & & $\mathrm{~B}_{1}$ & $\mathrm{~B}_{2}$ & $\mathrm{~B}_{3}$ \\
\hline$X(\mathrm{~mm})$ & 0 & 1000 & 1000 & 0 & $X^{\prime}(\mathrm{mm})$ & -50 & 50 & 50 \\
$Y(\mathrm{~mm})$ & 0 & 0 & 1000 & 1000 & $Y^{\prime}(\mathrm{mm})$ & -100 & -100 & -50 \\
$Z(\mathrm{~mm})$ & 1000 & 1000 & 1000 & 1000 & $Z^{\prime}(\mathrm{mm})$ & 5 & 5 & 5 \\
\hline
\end{tabular}

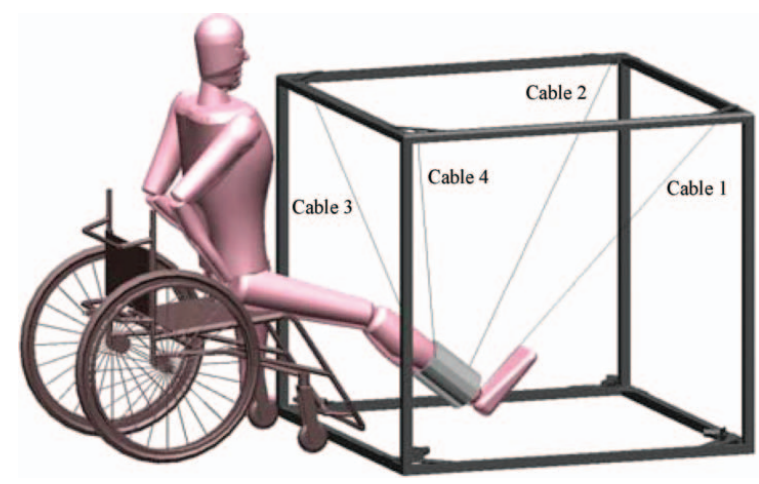

Figure 16. An overall ADAMS model of the manipulator and human body for the task as an aiding motion system for the lower limb movements.

The manipulator's end-effector has been considered attached to the leg and connected to cables in four distinct points.

Therefore, the configuration considered for the attachment points is shown in Figure 17(a). It is advantageous compared to the solution shown in Figure 17(b) as it prevents undesired and uncontrollable oscillations of the leg, as reported in Castelli et al. (2008). Depending on the position of the individual with respect to the robotic system and the size of the limb of the patient, the workspace of the limb can be identified. Within this area of work, the trajectory of the desired movement must be defined, similar to the phases described in the previous section.

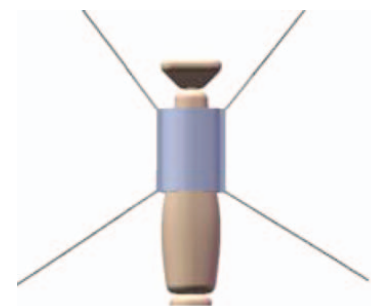

(a)

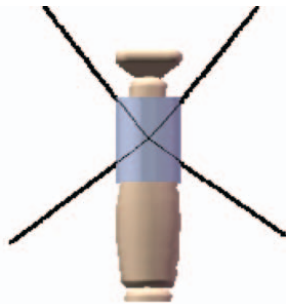

(a)
Figure 17. Two possible configurations for the end-effector attachment points (Castelli et al. 2008): (a) 4-4 configuration and (b) 4-1 configuration.
Figure 18(a) shows the path chosen for the model of the human body, which can be divided into three phases: (1) lift of the leg from point 1 to point 2 ; (2) adduction of the leg from point 2 to point 3 ; (3) abduction of the leg from point 3 to point 4 .

According to the above-mentioned leg movements in Figure 18(a), the outcome of the ADAMS simulation gives the law of variation of the cables' lengths for the movement shown in Figure 18(b).

Figure 19 shows numerical results obtained by a simulation implemented in the ADAMS environment for the

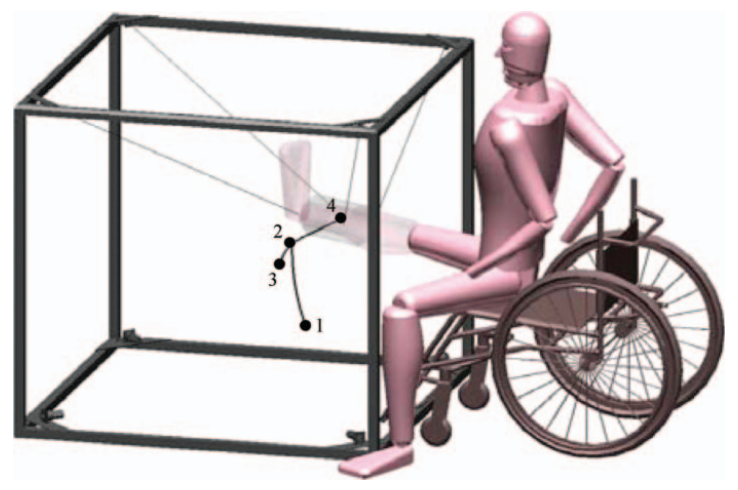

(a)

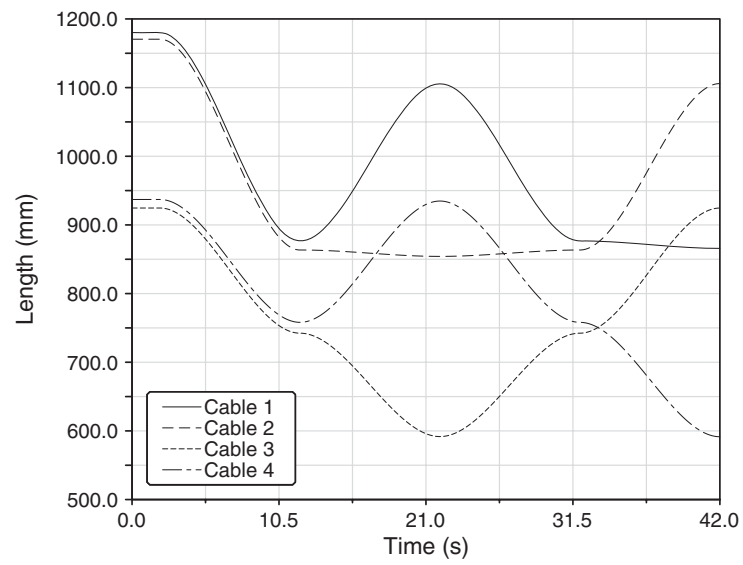

(b)

Figure 18. Desired trajectory for the lower limb movement: (a) 3D path and (b) variation of the cables' lengths. 


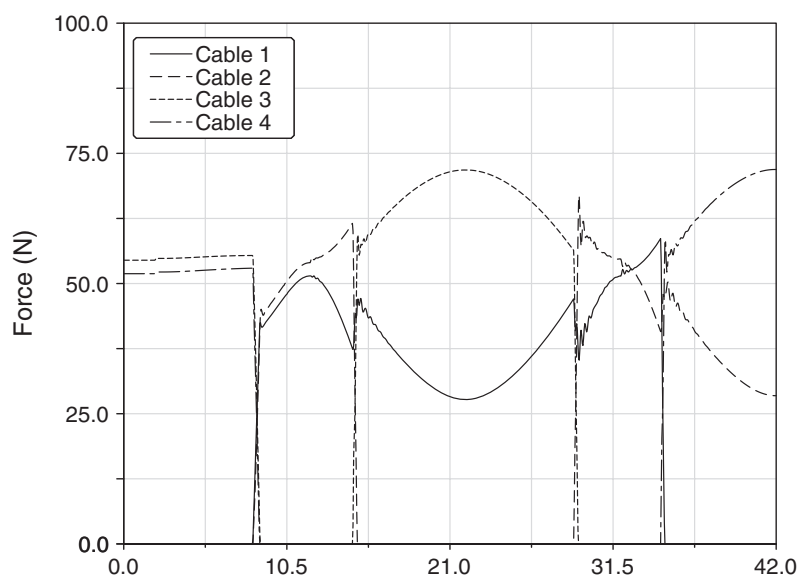

(a)

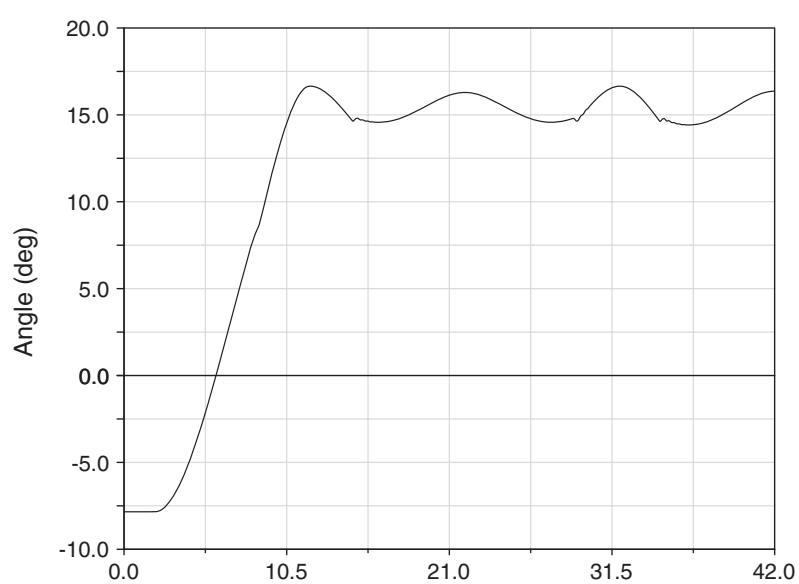

(b)

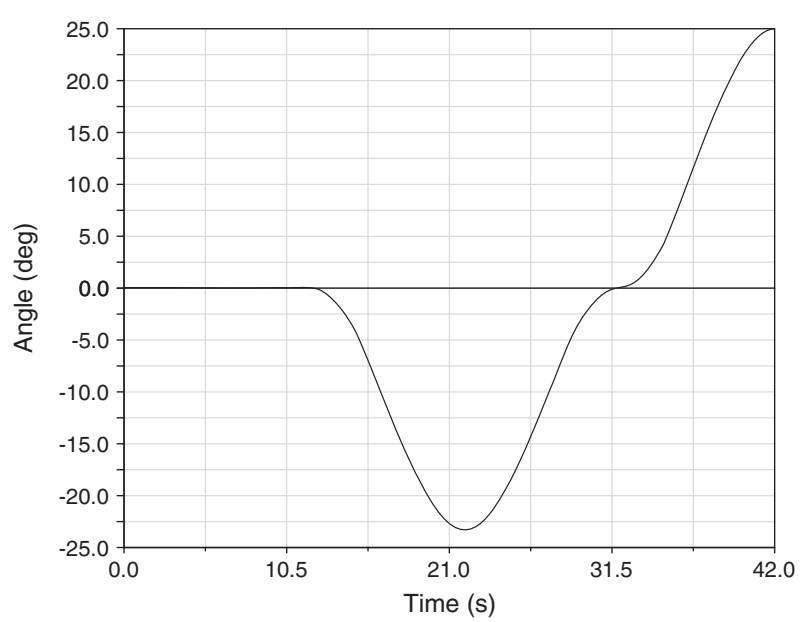

(c)

Figure 19. Simulation results for the movement in Figure 18 with a duration of $40 \mathrm{~s}$ : (a) cables' forces, (b) hip rotations for the movement of elevation, (c) adduction and abduction of the lower limb. proposed trajectory in Figure 18. In particular, Figure 19(a) shows the forces of the cables during the movement.

It is worth pointing out that during the movement cables 2 and 4 are almost always in tension. In Figures 19(b) and $19(\mathrm{c})$ rotations of the elevation hip, and adduction and abduction of the lower limb are shown.

\subsubsection{Experimental tests for the cable-based parallel ma- nipulator in the assisting motion application}

A system based on the CALOWI manipulator can also be considered for applications involving the movement of the lower limb. In particular, the laboratory experiments were carried out considering the manipulator in the spatial version with end-effector configuration 4-4 involving healthy adult volunteers. The layout of the experimental tests is shown in Figure 20.

Preliminary experiments were carried out programming the robotic system to perform the trajectory described in

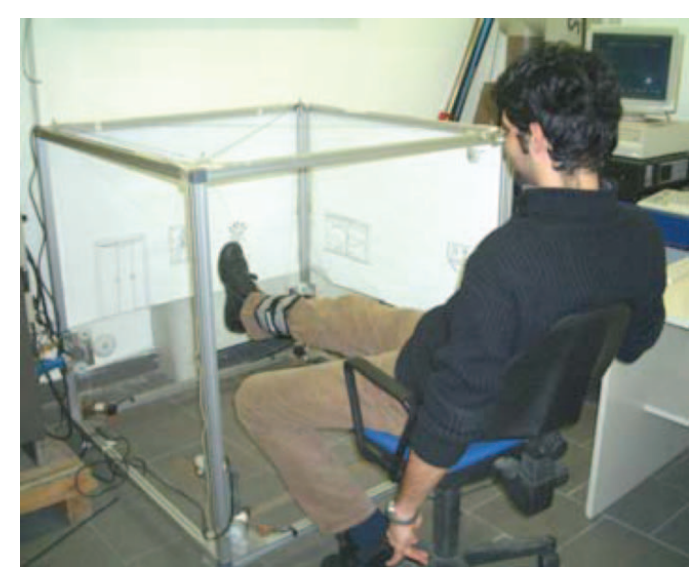

(a)

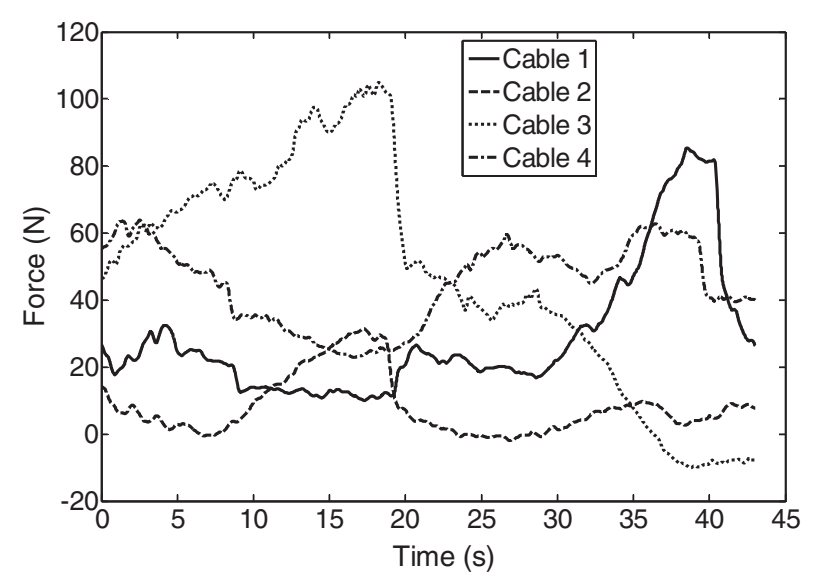

(b)

Figure 20. Layout for experimental tests using the CALOWI prototype for aiding the motion of the lower limb: (a) an experimental test and (b) measured cables' forces. 
Figure 18. Figure 20(b) shows experimental results of cables' forces, which have been obtained by considering only adult and healthy volunteers. It is worth noting that numerical results of Figure19 differ qualitatively substantially from those of Figure 20(b), although the magnitude of the maximum forces in the cable is the same. Such discrepancies are related to the assumptions for the development of the human body model, known to be very restrictive. Indeed, parts of the body and the end-effector have been considered as rigid bodies, but in reality they are deformable.

During the trials it was found that the characteristics of the human body tissues and end-effector are likely to greatly affect the simulation. In addition, there are also constraints in the joints of the human body that cannot allow the desired movement. Another factor of influence is the pose of the human body with respect to the manipulator. Indeed, the trajectory in Figure 18 is strictly related to the workspace of the lower limb for a specific body position. Therefore, for different poses of the human body in front of the manipulator, different behaviours of the manipulator and the human body can be obtained. To overcome this problem, a calibration of the system together with the individual can be carried out before trials. It should also be noted that physiological and neurological effects determine an incomplete passivity of the individual and a different interaction will be obtained.

Further development of the application will be carried out by considering a collaboration with medical personnel to study and interpret these physiological and neurological effects. In order to obtain consistent simulations, it is therefore necessary to refine the model of the human body, taking into consideration issues such as main characteristics of the tissues. It is also necessary to achieve a versatile positioning system, able to adapt the robotic system to any person, and able to accommodate patients placed in different configurations.

\section{Conclusions}

In this paper applications have been presented of a cablebased system as aiding and guiding motion devices. In particular, it has been shown that a reconfigurable four-cablebased manipulator can be used as a motion assistance device for guiding movements of the upper and lower limbs. In such applications, it is of crucial importance that the system, plan and simulate suitable paths. Therefore, models have been developed and simulations have been carried out in the ADAMS environment for the above-mentioned tasks. Simulations were carried out by using a Pentium with $3 \mathrm{GHz}$, and each simulation required less than 1 minute. Experimental tests have been carried out to verify the feasibility of the proposed solution. The mechanical design of a real scale prototype for the assisting device is under development. It is worth mentioning that a four-cable reconfigurable parallel manipulator can be effectively used for upper and lower limb guidance by simply reconfiguring the system.

\section{References}

Beyl P, Van Damme M, Van Ham R, Vanderborght B, Lefeber D. 2009. Design and control of a lower limb exoskeleton for robot-assisted gait training. J Appl Bionics Biomech. 6(2):229-243.

Castelli G. 2010. Analysis, modeling and experimentation of cable-based manipulators [PhD thesis]. [Cassino]: University of Cassino.

Castelli G, Ottaviano E. 2009. Modelling and simulation of a cable-based parallel manipulator as an assisting device. Proceedings of the 5thInternational Workshop on Computational Kinematics CK 2009, Duisburg, ISBN: 978-3-642-01946-3, pp. 17-24.

Castelli G, Ottaviano E, Ceccarelli M. 2008. Modelling and simulation of a cable-based manipulator for rehabilitation therapies. Proceedings of the 3rd International Conference on Optimization of the Robots and Manipulators, Predeal, pp. 277-282.

Chugo D, Asawa T, Kitamura T, Jia S, Takase K. 2008. A rehabilitation walker with standing and walking assistance. Proceedings of the International Conference on Intelligent Robots and Systems, Nice, pp. 260-265.

Dubowsky S, Genot F, Godding S, Kozono H, Skwersky A, Yu H, Yu LS. 2000. PAMM - A robotic aid to the elderly for mobility assistance and monitoring. IEEE International Conference on Robotics and Automation, San Francisco, Vol. 1, pp. 570-576.

Gray H. 1918. Anatomy of the human body. New York, NY: Lea \& Febiger.

Hiller M, Fang S, Mielczarek S, Verhoeven R, Franitza D. 2005. Design, analysis and realization of tendon-based parallel manipulators. Mech Mach Theory. 40:429-445.

Kiguchi K, Fukuda T. 2004. A 3 DOF exoskeleton for upperlimb motion assist: consideration of the effect of bi-articular muscles. IEEE International Conference on Robotics and $\mathrm{Au}-$ tomation, pp. 2424-2429.

Marlene JA, Cooper J. 1995. Biomechanics of human movement. Madison (WI): Brown \& Benchmark.

Mayhem D, Bachrach B, Rymer WZ, Beer RF. 2005. Development of the MACARM - a novel cable robot for upper limb neurorehabilitation. IEEE 9th International Conference on Rehabilitation Robotics, Chicago, Vol. 1, pp. 299-302.

Médéric P, Pasqui V, Plumet F, Bidaud P. 2004. Design of a walking-aid and sit to stand transfer assisting device for elderly people. 15 th CISM-IFToMM Symposium on Robot Design, Dynamics and Control, St Hubert.

Ottaviano E. 2008. Design issues and application of cable-based parallel manipulators for rehabilitation therapy. J Appl Bionics Biomech. 5(2):65-75.

Ottaviano E, Castelli G, Cannella G. 2008. A cable-based system for aiding elderly people in sit to stand transfer. Mech Based Design Struct Mach. 36(4):1-20.

Riechel AT, Ebert-Uphoff I. 2004. Force-feasible workspace analysis for underconstrained, point-mass cable robots. IEEE International Conference on Robotics and Automation, New Orleans, pp. 4956-4962.

Roberts RG, Graham T, Lippitt T. 1998. On the inverse kinematics, statics and fault tolerance of cable-suspended robots. J. Robotic Syst. 15(10):581-597.

Rosati G, Zanotto D, Secoli R, Rossi A. 2009. Design and control of two planar cable-driven robots for upper-limb neurorehabilitation. 2009 IEEE 11th International Conference on Rehabilitation Robotics, Kyoto, pp. 560-565. 
Stienen AHA, Hekman EEG, Schouten AC, van der Helm FCT, van der Kooij H. 2009. Suitability of hydraulic disk brakes for passive actuation of upper-extremity rehabilitation exoskeleton. J Appl Bionics Biomech. 6(2): 103-114.

Sugar TG, Koeneman EJ, Herman JB, Huang R, Schultz H, Herring RS, Wanberg DE, Balasubramanian J, Swenson S, Ward P. 2007. Design and control of RUPERT: a device for robotic upper extremity repetitive therapy. IEEE Trans Neural Syst Rehab Eng. 15(3):336-346.

Verhoeven R, Hiller M, Tadokoro S. 1998. Workspace, stiffness, singularities and classification of tendon-driven Stewartplatforms. International Symposium on Advances in Robot Kinematics, Strobl (Austria), pp. 105-114.

Winter, DA. 1990. Biomechanics and motor control of human movement. New York, NY: Wiley. 

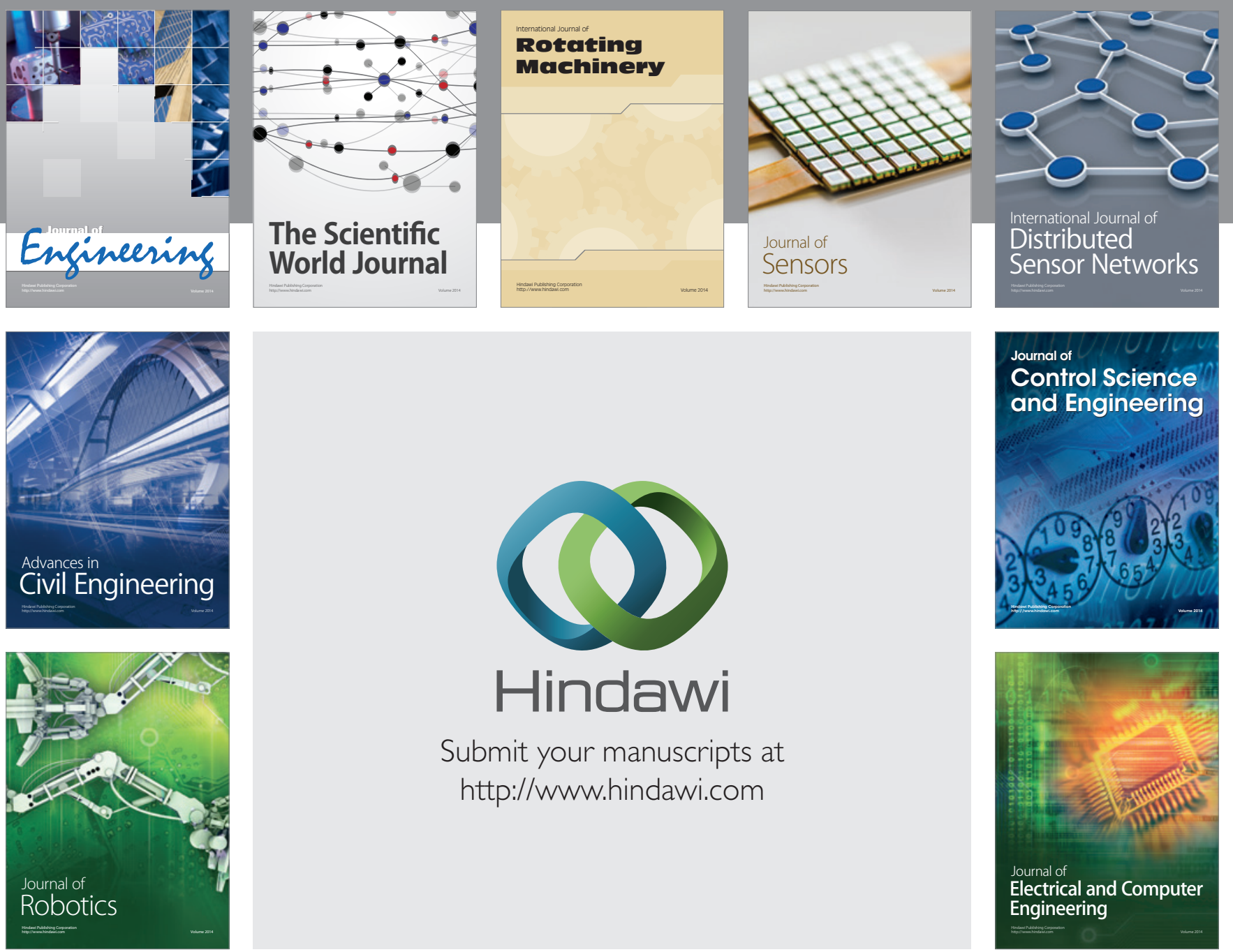

Submit your manuscripts at

http://www.hindawi.com
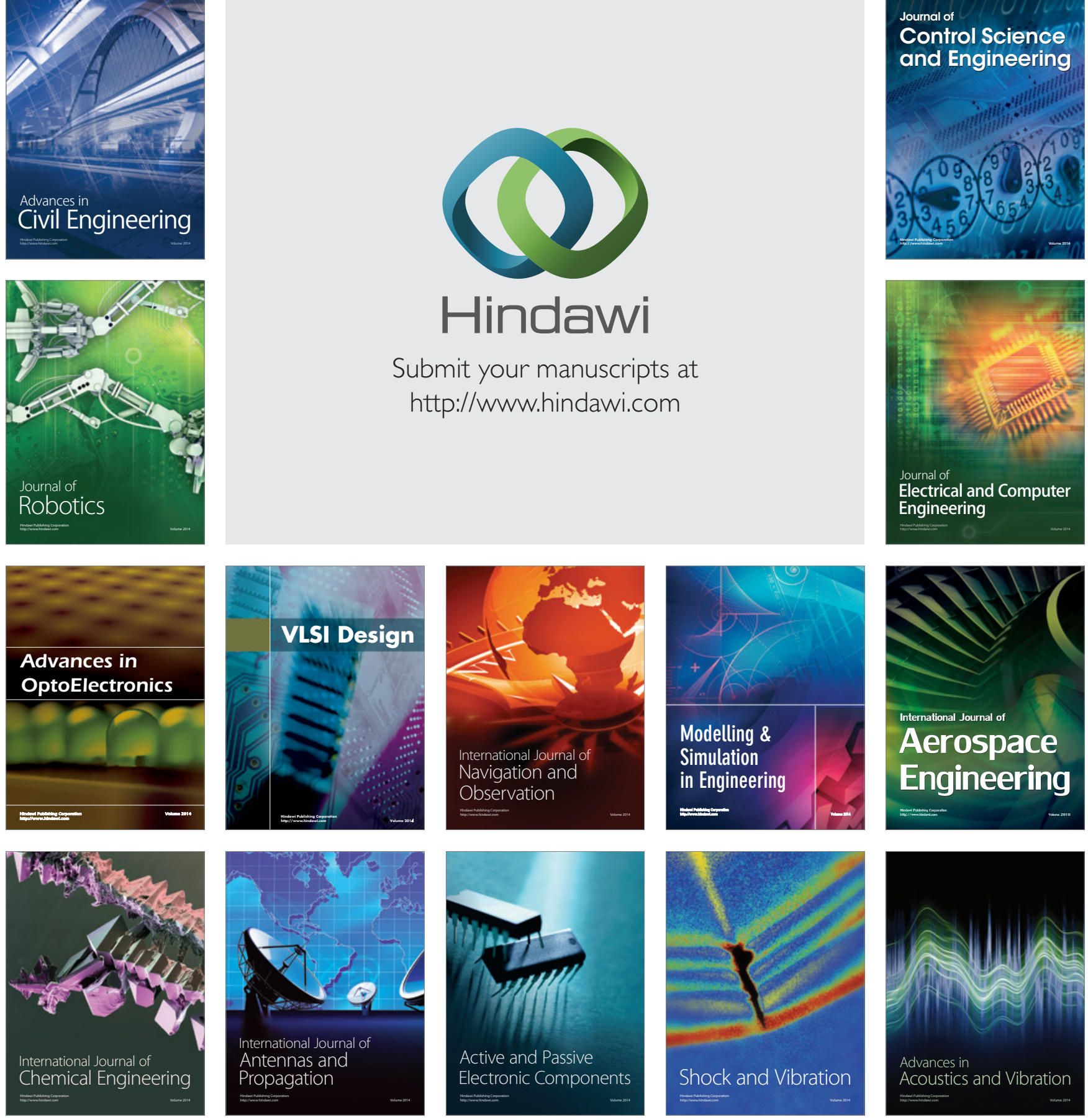